\title{
Comparison of Edge and Internal Transport Barriers in Drift Wave Predictive Simulations
}

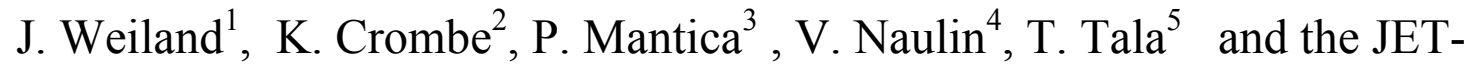 \\ EFDA Contributors*
}

JET-EFDA, Culham Science Centre, OX14 3DB, Abingdon, UK

1. Department of Earth and Space Sciences, Chalmers. University of Technology and Euratom-VR Association, S41296 Gothenburg, Sweden

2. Association Euratom-Belgian State Department of Applied Physics, Ghent University, Rozier 44 B-9000 Ghent

Belgium

3. Istituto di Fisica del Plasma CNR-EURATOM, via Cozzi 53, 20125 Milano, Italy

4. Association Euratom-Risø DTU, Denmark

5. Association Euratom-Tekes, VTT, P.O. Box 1000, FIN-02044 VTT, Finland

*See the Appendix of F. Romanelli et al., Proceedings of the $23^{\text {rd }}$ IAEA Fusion Energy Conference 2010, Daejon, Korea

PACS: 52.55-s, 52.30.-q, 52.35-Ra

\begin{abstract}
We have simulated the formation of an internal transport barrier on JET including a self-consistent treatment of ion and electron temperatures and poloidal and toroidal momentum.

Similar simulations of edge transport barriers, including the L-H transition have also been made. However, here only polodal momentum and the temperatures were simulated. The internal barrier included an anomalous spinup of poloidal momentum similar to that in the experiment. Also the edge barrier was accompanied by a spinup of poloidal momentum. The experimental density (with no barrier) was used and kept fixed for the internal barrier. For the edge barrier the edge density was varied and it turned out that a lower edge density gave a stronger barrier. Electromagnetic and nonlocal effects were important for both types of barriers.
\end{abstract}

\section{INTRODUCTION}

The understanding of the formation of transport barriers is presently one of the outstanding issues in fusion transport research ${ }^{1-8}$. This problem area consists of several parts including the separate dynamics of poloidal and toroidal momentum, effects of field ripple at the edge, magnetic q profile and several other issues. The most striking new feature observed is that of the poloidal momentum spinup ${ }^{3}$. This has been found to give rotation of up to an order of magnitude larger than the neoclassical rotation. For the internal barrier, such poloidal spinup, requires a poloidal momentum pinch and is due to Zonal flows generated by drift wave turbulence. Results consistent with the JET barrier pulses 58094 and 69454 have been obtained but similar spinup was also obtained for the barrier shot 51976 (no experimental poloidal rotation measurements available). The edge barrier has only been included quite recently by reducing the edge temperatures by a factor 5 . This leads to the formation of a barrier in the simulations which essentially restores the experimental temperatures at the top of the barriers. Several similarities between internal and edge barriers were found.

\section{FORMULATION}

For the toroidal momentum transport we use the recently derived toroidal symmetry breaking effects ${ }^{5-7}$. Adding electromagnetic effects to the formulation in Ref 7 we get:

$$
m_{i} N_{i}\left(\frac{\partial}{\partial t}+2 \vec{U}_{D i} \cdot \vec{\nabla}\right) \delta u_{\|}=-m_{i} N_{i} \overrightarrow{\mathrm{u}}_{E} \cdot \nabla U_{\| 0}-\left[\widehat{e}_{\|} \cdot \nabla+U_{\| 0} \frac{m_{i} \vec{U}_{D_{i}}}{T_{i}} \cdot \nabla\right]\left(\delta p_{i}+e N_{i} \phi-\frac{\omega+\omega_{* e}\left(1+\eta_{e}\right) / \tau}{k_{\|} c} A_{\|}\right)
$$

IFP-CNR - Chalmers Workshop on Nonlinear Phenomena in Fusion Plasmas

AIP Conf. Proc. 1392, 85-91 (2011); doi: 10.1063/1.3647234

(C) 2011 American Institute of Physics 978-0-7354-0962-0/ $\$ 30.00$ 


$$
\begin{gathered}
\delta u_{\|}=-\frac{k_{\theta} D_{B}}{\omega-2 \omega_{D i}} \frac{d U_{\| 0}}{d r}+\frac{<k_{\|}>+\omega_{D i} U_{\| 0} /\left(\tau \cdot c_{s}^{2}\right)}{\omega-2 \omega_{D i}}\left(\delta p+n e \phi-\frac{\omega+\omega_{* e}\left(1+\eta_{e}\right) / \tau}{k_{\|} c} A_{\|}\right) /\left(m_{i} n_{i}\right) \\
\vec{U}_{D i}=2 \frac{c T_{i}}{e B^{2}} \widehat{n} \times \nabla B ; \quad \Gamma_{\|}=<\mathrm{v}_{\mathrm{Er}} \delta \mathrm{u}_{\|}>\approx \Gamma_{\phi}
\end{gathered}
$$

Here the convective magnetic drift term in the left hand side can either be obtained from a gyrofluid approach ${ }^{6,8}$ or from fluid equations including the stress tensor ${ }^{6,7}$. It was recently found that electromagnetic effects increase the toroidal momentum pinch ${ }^{9}$. The first term on the right hand side is the ExB convection in the background velocity gradient. This gives the diagonal element. Using the saturation level:

$$
\frac{e \phi}{T_{e}}=\frac{\gamma}{k_{\theta} c_{s} k_{r} \rho}=\frac{\gamma}{\omega_{* e}} \frac{1}{k_{r} L_{n}}
$$

we then get the diagonal element :

$$
\chi_{\phi}=\frac{\gamma^{3} / k^{2}}{\left(\omega_{r}-2 \omega_{D i}\right)^{2}+\gamma^{2}}
$$

It is important to note, however, that this diagonal element is not uniquely defined.

The reason is that it depends on the frequency which, in turn, depends on all other gradients in the system through the dispersion relation.

The poloidal flux is given by the Reynolds stress as:

$$
\Gamma_{p}=<\mathrm{v}_{\mathrm{Er}} \mathrm{v}_{\theta}>=-D_{B}{ }^{2} k_{r} k_{\theta} \frac{1}{2} \widehat{\phi}^{*}\left[\widehat{\phi}+\frac{1}{\tau} \hat{P}_{i}\right]+c . C
$$

Here also the diamagnetic drift was included as a convected velocity

\section{SIMULATIONS}

We have simulated the JET shots 58094, 51976 and 69454. All of these give a strong spinup of the poloidal rotation in the simulations. However experimental data on poloidal rotation is missing for 51976 . We here show the barrier formation in JET69454.

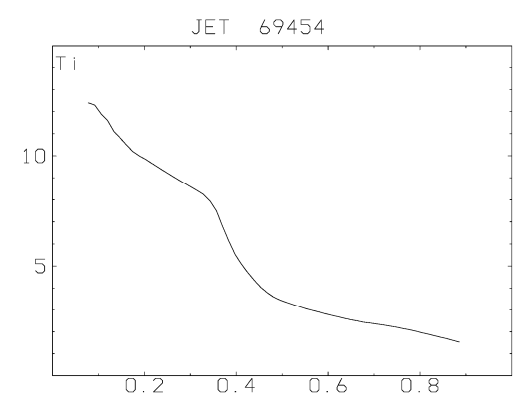

Fig 1a Experimental Ti



Fig 1b Experimental Vtor 


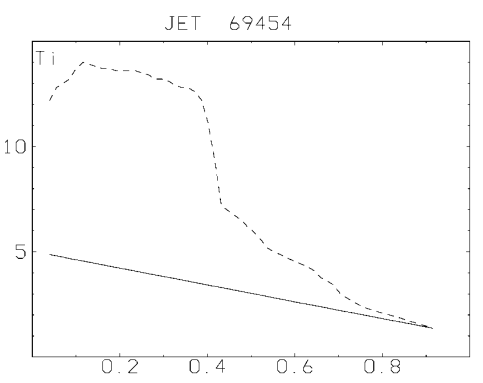

Fig 2a Simulated Ti (dotted) Initial profile (full )



Fig 2b Simulated Vtor (dotted) Initial profile (full)

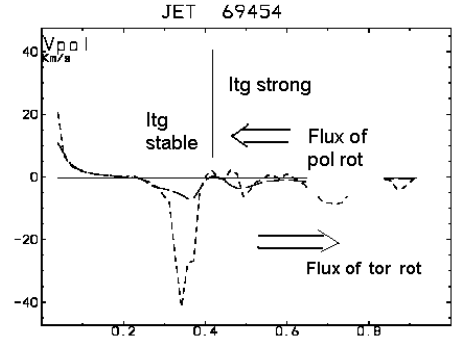

Fig 2c Simulated poloidal spinup (dotted)

Neoclassical rotation (dashed)

Initial condition (full)

The experimental poloidal spinup has the same location and magnitude $(40 \mathrm{~km} / \mathrm{s})$ but was measured on impurities and therefore had the opposite direction in this case. The net poloidal flux is indicated as inward in the picture but is in the end zero when it is balanced by outward flux from the enhanced piled up rotation since there is no poloidal momentum source. The net toroidal momentum flux is outward in equilibrium since there is a torque deposition in the interior. There is, however, a strong inward component of the toroidal flux which enhances the inward peaking. We notice that the simulated profiles have somewhat more pronounced barriers (in particular Vtor) but the central values are quite similar to the experimental. There was no trace of the barrier in the initial conditions. The ITG mode is stable within the barrier while the Trapped electron mode is marginally unstable. The toroidal rotation continues to grow towards the axis inside the barrier because of the torque deposition. When there is no barrier there is no poloidal spinup. In these cases the poloidal momentum flux fluctuates in both time and space. The location of the barrier is due to a combination of small magnetic shear and the power deposition profile.

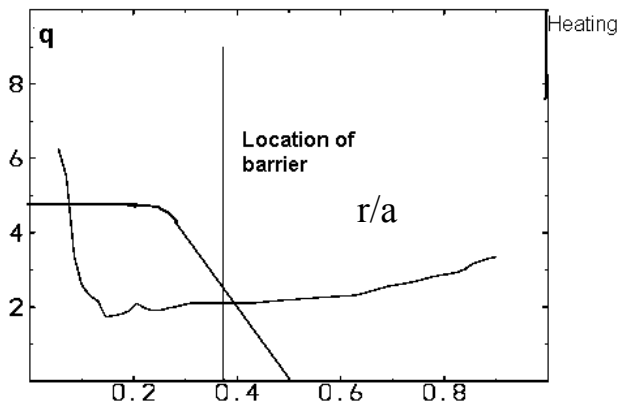

Fig 3 Picture showing that the main power deposition is inside the location of the barrier (at 0.4) where magnetic shear is still small. 


\section{ELECTROMAGNETIC EFFECTS}

We recently discovered that electromagnetic effects have a significant influence on the momentum transport. This enters through the toroidal momentum ${ }^{9}$ but also influences poloidal momentum. In fact, there is no pronounced barrier and no poloidal spinup in the electrostatic limit.

Fig 4a Electrostatic Pol rot

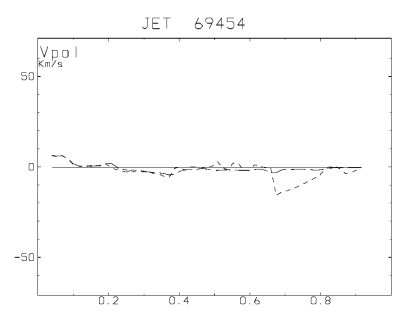

Fig $4 \mathrm{~b}$ Electrostatic Tor. rot

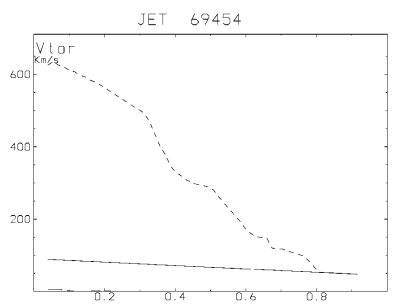

\section{EDGE BARRIER}

The present model contains also the main physics ingredients for the $\mathrm{L}-\mathrm{H}$ transition ${ }^{10}$. We have here added collisions also on free electrons and a Kink driving term which causes peeling.

In fact already our first transport code from 1991 gave an L-H transition due to diamagnetic stabilization of the main drift waves ${ }^{11}$. This model did not include collisions and was clearly not applicable at the edge. However, trapped electrons have the same description as electrons where the parallel motion is completely impeded by collisions. In this case a new type of reactive resistive ballooning mode was found by Novakovskii et.al ${ }^{12}$ and this mode was also included in turbulence simulations ${ }^{13}$. This mode has several features in common with our drift wave model and, in fact, the systems can be combined by using a complex trapped fraction i.e. an adiabaticity index ${ }^{14}$. All these systems ${ }^{11-14}$ include a possibility for diamagnetic stabilization of a strong instability in L-mode which could correspond to a L-H transition. The systems in Refs 11 and 15 also include a condensation mode in H-mode which gives a particle pinch on the edge barrier. Such a diamagnetic stabilization was included also in the turbulence simulations in Ref 13 which were in fairly good agreement with experiment ${ }^{16}$ although only in the local sense.. While our first observation of an L-H transition in transport simulations ${ }^{11}$ gave edge barriers in both temperature and density, we have in the present work only simulated the formation of temperature barriers, keeping density fixed. However, we have varied the density at the edge. Our first simulation of a scan in heating also gave the right power degradation of the confinementtime, i.e. $\tau_{\mathrm{E}} \sim \mathrm{P}^{-2 / 3}$ where $\mathrm{P}$ is the heating power and the increase in the confinement time at the L-H transition was a factor 2.5 . Thus the drift wave power scaling and the difference between $\mathrm{L}$ and $\mathrm{H}$ mode equilibria in the core were in agreement with experiment although the H-mode barrier was not resolved. This first simulation of the L-H transition did not contain flowshear at all thus the L-H transition was entirely diamagnetic. In the present work we have used the same model as for the internal barriers except that we kept the toroidal momentum fixed. We also added collisions on free electrons and the kink driving term in $\mathrm{Eq}(6)$. Furthermore we have used a varying correlation length for electron modes

$$
\left(\mathrm{k}_{\theta} \rho_{\mathrm{s}}\right)_{e}=0.024-0.0064\left(\widehat{v}_{e i}-0.61\right)+0.4 \beta_{e}
$$

as given by Eq (5) where $\widehat{v}_{e i}$ is the electron ion collision frequency normalized by the electron magnetic driftrequency. This correlation length, which has been used for some time, corrects the previously too small electron transport and now gives $\mathrm{k}_{\theta} \rho \approx 0.1$ at the edge. It was derived using the trapped fraction 0.5 but is rather insensitive to this choice. Thus we may ignore this dependence for typical trapped fractions in a tokamak. We now use the equations:

$$
\frac{\partial n_{e f}}{\partial t}+\nabla \cdot\left[n_{e f}\left(\mathbf{v}_{\mathbf{E}}+\mathbf{v}_{* \mathbf{e}}+\mathrm{v}_{\| 0} \frac{\delta \mathbf{B}_{\perp}}{\mathrm{B}}+\mathrm{v}_{\|} \widehat{\mathbf{e}}_{\|}\right)\right]=0
$$




$$
\frac{\partial n_{e f}}{\partial t}=\frac{e}{T_{e}}\left(\phi+\frac{\omega_{* e}-\omega}{k_{\|}} A_{\|}\right)+i \frac{\mathrm{v}_{\| \mathrm{e}}}{\mathrm{k}_{\|} D_{e}}
$$

From which:

$$
\frac{e A_{\|}}{T_{e}}=k_{\|} \frac{\omega-\omega_{* e}}{\omega\left(\omega-\omega_{* e}\right)+\omega_{D e}\left(\omega_{* e T}-\omega\right)-\frac{k_{\|} k_{\theta} T_{e}}{e^{2} B n_{0}} \frac{\partial \mathrm{J}_{\| 0}}{\partial \mathrm{r}}-\mathrm{k}_{\perp}{ }^{2} \rho_{s}{ }^{2} k_{\|}{ }^{2} \mathrm{v}_{\mathrm{A}}{ }^{2}(1-i \delta)} \frac{e \phi}{T_{e}}
$$

where

$$
\delta=\frac{\omega-\omega_{D e}}{k_{\|}^{2} D_{e}} \quad D_{e}=\frac{T_{e}}{m_{e} v_{e}}
$$

\section{SIMULATIONS}

We will now show simulations of edge barriers where the basic data are taken from JET 69454. However, we have varied the outer boundaries and the poloidal magnetic field by varying $\mathrm{q}$.



Start profile

Fig 5. Edge barrier for JET 69454. The initial edge temperature has been reduced from 1.4 to $0.2 \mathrm{KeV}$. Here the experimental edge density was used. We notice that the L-H transition in the simulation is very close to restoring the experimental edge temperature at the top of the pedestal.

This barrier is here caused by poloidal momentum spinup as shown in Fig 6 .

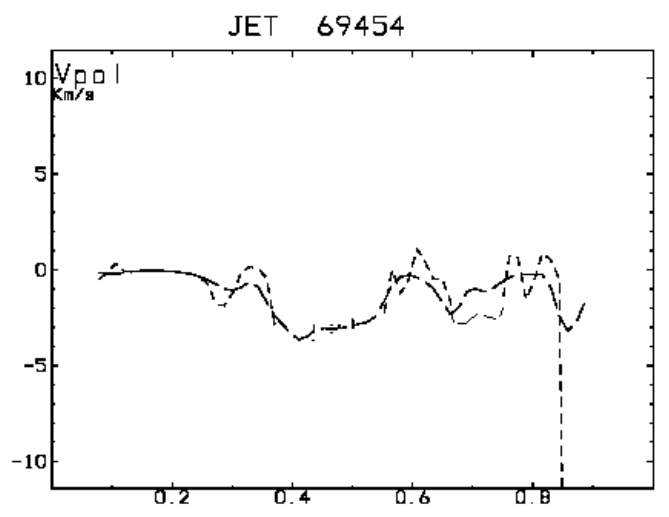

Fig 6 Poloidal rotation in a shot with H-mode barrier 
The edge barrier increases if we increase $B_{p}$ and/or reduce the edge density.



Fig 7. The same parameters as in Fig 5 except for a $50 \%$ increase in $\mathrm{B}_{\mathrm{p}}$ and a $50 \%$ reduction of the edge density .

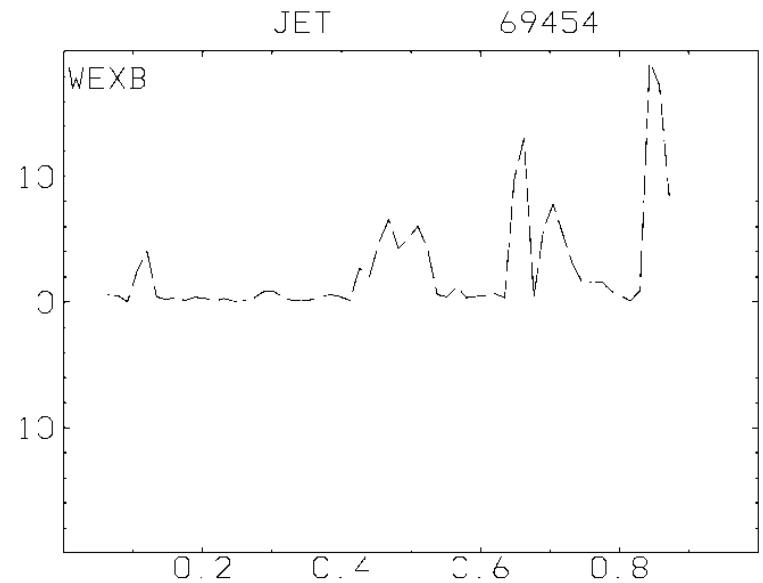

Fig 8 Profile of flowshear corresponding to the simulation in Fig 7.

\section{PEELING}

When we increase $B_{p}$ but keep the density fixed in the case shown in Fig 5 we get peeling.

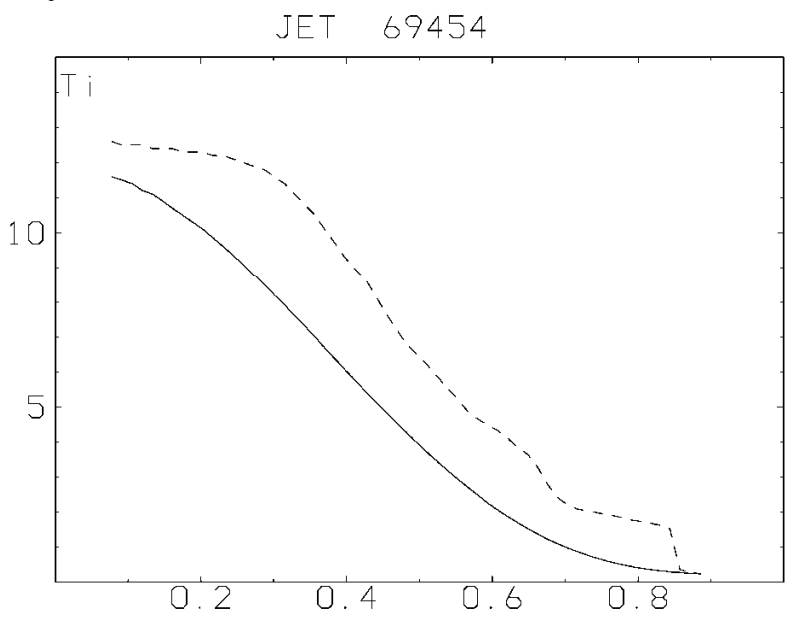

Fig 9 Simulation showing the effect of the kink term. In this case the outer part of the barrier is peeled off. Data as in fig 5 but with a $50 \%$ increase in $\mathrm{B}_{\mathrm{p}}$. 


\section{DISCUSSION}

We have shown how a fluid model containing both poloidal and toroidal momentum transport can describe the formation of a transport barrier in a selfconsistent simulation of four channels, ion and electron temperature and poloidal and toroidal momenta. The poloidal spinup has previously been recovered also for JET51976 and JET58094 although experimental measurements of the poloidal rotation are missing for 51976. The barrier location is here a result of small magnetic shear (optimized shear) and the power deposition. Rotation is driven by the temperature scale length and this requires both large thermal flux and an additional mechanism which limits transport so that the scale length is reduced. The Trapped Electron mode dominates transport in the whole region of small and negative magnetic shear. However, the ITG mode would also be unstable in the absence of flowshear. Since this model does not include transport due to perturbed magnetic flux surfaces, it is not sensitive to exact values of magnetic $\mathrm{q}$ (like rationals). The convergence of the results with respect to resolution has been tested with almost the same results between 50 and 99 radial gridpoints. We have also tested results in the electrostatic limit and in the absence of electron trapping. No internal barrier is formed in these cases. In particular electromagnetic effects have recently been found to be important for the toroidal momentum pinch ${ }^{9}$. This opens up a possible explanation for the stronger barrier in the simulation. The model for elongation is rather crude and usually underestimates the effect. Elongation acts as to reduce electromagnetic effects which, in turn, tend to increase the toroidal momentum pinch. Thus a stronger effect of elongation is expected to reduce the momentum pinch. We have here used a separate correlation length for electron modes (5) using the same method as in Ref 17. This method has recently been successful in calculating the correlation length in the presence of flowshear ${ }^{18}$. We have made scaling of the edge barrier with edge density and $\mathrm{B}_{\mathrm{p}}$. The hight of the barrier is increased when the density is reduced (a factor 0.5 in density increases the edge barrier height by about $15 \%$ ) and also increased when $B_{p}$ is increased but this effect is even less sensitive. Peeling is also more effective for large $B_{p}$. This is natural since $B_{p}$ is directly linked to the background current.

\section{REFERENCES}

1. J.E. Rice, E.S. Marmar, F. Bombarda and L. Qu, Nuclear Fusion 37, 421 (1997).

2. P.C. deVries, K.M. Rantamäki, C. Giroud, E. Asp, G. Corrigan, A. Eriksson, M. deGreef, I. Jenkins, H.C.M. Knoops, P. Mantica, H. Nordman, P. Strand, T. TALA, J. Weiland, K-D Zastrow and the JET-EFDA contributors, Plasma Phys. Control. Fusion 48, 1693 (2006).

3. K. Crombe, Y. Andrew, M. Brix et. al. Phys. Rev. Lett. 95, 155003 (2005).

4. T. Tala, K-D. Zastrow, J. Ferreira et.al. Phys. Rev. Lett 102, 075001 (2009).

5. T.S. Hahm, P.H. Diamond, O.D. Gurcan and G. Rewoldt, Phys. Plasmas 14, 072302-1 (2007).

6. D. Strinzi, A.G. Peeters and J. Weiland, Phys. Plasmas 15, 044502 (2008).

7. J. Weiland, R. Singh, H. Nordman. P.K. Kaw, A. Peeters and D. Strintzi Nuclear Fusion 49, 065033 (2009)).

8. R.E. Waltz, R.R. Dominguez and G.W. Hammett, Phys. Fluids B4, 3138 (1992).

9. A. Mahmood, A. Eriksson and J. Weiland, Electromagnetic effects on toroidal momentum transport, Physics of Plasmas 17, 122310 (2010).

10. F. Wagner, et. al. Phys. Rev. Lett. 53, 1453 (1982).

11. J. Weiland and H. Nordman, Nucl. Fusion 31, 390 (1991).

12. S. Novakovskii, P. Guzdar, J. Drake, C.S. Liu and F. Waelbroeck, Phys. Plasmas 2, 3764 (1995).

13. B.N. Rogers, J. Drake and A. Zeiler, Phys. Rev. Lett. 81, 4396 (1998).

14. R. Moestam and J. Weiland, Nuclear Fusion 42, 663 (2002).

15. R. Singh and J. Weiland, Phys. Plasmas 6, 1397 (1999).

16. A.E. Hubbard, R.L. Boivin, J.F. Drake, M. Greenwald, Y. In, J.H. Irby, B.N. Rogers and J.A. Snipes, Plasma Phys. Control Fusion 40, 689 (1998).

17. J. Weiland and I. Holod, Phys. Plasmas 12, 012505 (2005).

18. J. Weiland and P. Mantica, et.al. Effects of flow shear on the correlation length of drift wave turbulence, Proc. $38^{\text {th }}$ EPS Conference on Plasama Physics, Strasbourg, June 27-July 1 2011, Paper P5.130. 